\title{
Anuros do cerrado da Estação Ecológica e da Floresta Estadual de Assis, sudeste do Brasil
}

\author{
José Wagner Ribeiro-Júnior ${ }^{1}$ \& Jaime Bertoluci ${ }^{2,3}$ \\ ${ }^{1}$ Coordenação de Pesquisas em Ecologia, Instituto Nacional de Pesquisas da Amazônia, \\ CP 478, CEP 69011-970, Manaus, AM, Brasil, http://www.inpa.gov.br/ \\ ${ }^{2}$ Departamento de Ciências Biológicas, Escola Superior de Agricultura Luiz, de Queiroz, \\ Universidade de São Paulo - USP \\ Av. Pádua Dias 11, CEP 13418-900, Piracicaba, SP, Brasil \\ ${ }^{3}$ Autor para correspondência: Jaime Bertoluci,e-mail: bertoluc@esalq.usp.br, http://www.esalq.usp.br/
}

RIBEIRO-JÚNIOR, J.W. \& BERTOLUCI, J. Anurans of the cerrado of the Estação Ecológica and the Floresta Estadual de Assis, southeastern Brazil. Biota Neotrop., 9(1): http://www.biotaneotropica.org.br/v9n1/en/abst ract?inventory+bn02709012009.

Abstract: The Brazilian cerrado is a hotspot of biodiversity, areas with many endemic species subjected to severe habitat loss. About 150 amphibian species are known to the Brazilian cerrado, and $28 \%$ of them are endemic. Studies focusing cerrado anuran communities are rare. Here we provide a commented list of anurans of two conservation unities of the municipality of Assis, southeastern Brazil: the Estação Ecológica de Assis and the Floresta Estadual de Assis. Twenty three anuran species were recorded, and these belong to 13 genera and six families. The anuran fauna of the cerrado of Assis is dominated by the families Hylidae (eight species) and Leptodactylidae (six species), a pattern commonly found in neotropical sites. Species richness and taxonomic composition at the family level were similar to those of other Brazilian cerrados and perturbed areas characterized by deforestation and increase of open areas. The taxocenosis contains species widespread in open formations of the Cerrado-Caatinga-Chaco complex.

Keywords: amphibians, anurans, cerrado, inventory.

RIBEIRO-JÚNIOR, J.W. \& BERTOLUCI, J. Anuros do cerrado da Estação Ecológica e da Floresta Estadual de Assis, sudeste do Brasil. Biota Neotrop., 9(1): http://www.biotaneotropica.org.br/v9n1/pt/abstract?invento ry+bn02709012009.

Resumo: O cerrado brasileiro é um dos 25 "hotspots" da Terra, áreas caracterizadas pela concentração de espécies endêmicas e por experimentar excepcional perda de hábitat. São conhecidas cerca de 150 espécies de anfíbios no Cerrado brasileiro, e aproximadamente $28 \%$ dessas espécies são endêmicas. Trabalhos com comunidades de anuros do cerrado ainda são escassos, e a forte pressão antrópica, o alto grau de endemismo e o declínio de populações de anuros registrados nesse bioma reforçam a importância de estudos relacionados à anurofauna. O presente trabalho apresenta um inventário de espécies de anuros em duas unidades de conservação no cerrado da cidade de Assis, sudoeste do Estado de São Paulo: Estação Ecológica de Assis e Floresta Estadual de Assis. Durante o estudo, foram registradas 23 espécies distribuídas em 13 gêneros e seis famílias. A fauna de anuros do cerrado de Assis é fortemente dominada pelas famílias Hylidae (oito espécies) e Leptodactylidae (seis espécies), um padrão comumente encontrado em localidades da região Neotropical. A riqueza de espécies e a representatividade das famílias foram similares às encontradas em outras áreas de cerrado do Brasil e de formações vegetais antropizadas com perda de área florestal e aumento de áreas abertas. A taxocenose de anuros da localidade de estudo é composta por espécies amplamente distribuídas em ambientes abertos do complexo Cerrado-Caatinga-Chaco.

Palavras-chave: anfíbios, anuros, cerrado, inventário. 


\section{Introdução}

O cerrado é o segundo maior bioma brasileiro em extensão, com mais de $2.000 .000 \mathrm{~km}^{2}$ (Ratter et al. 1997). Nos últimos 35 anos, mais da metade da área do cerrado foi transformada em pastagens, plantações de grãos e outros tipos de uso (Klink \& Machado 2005), sendo que as unidades de conservação cobrem menos de $2 \%$ de sua área total (Ratter et al. 1997). O Estado de São Paulo, que possuía originalmente cerca de $34.700 \mathrm{~km}^{2}$ de Cerrado (14\% de sua área total), encontra-se em situação mais grave, já que os remanescentes dessa vegetação cobrem menos de $1 \%$ do Estado e apenas cerca de metade do que restou encontra-se protegida na forma de unidades de conservação (Bitencourt \& Mendonça 2004). Segundo Myers et al. (2005), o cerrado brasileiro é um dos 25 "hotspots" da Terra, áreas caracterizadas pela concentração de espécies endêmicas e por experimentar excepcional perda de hábitat.

São conhecidas pelo menos 150 espécies de anfíbios no Cerrado, e aproximadamente $28 \%$ dessas espécies são endêmicas desse bioma (Klink \& Machado 2005). Poucos trabalhos tratam das taxocenoses de anuros do cerrado, destacando-se aqueles desenvolvidos na Serra da Canastra, no município de João Pinheiro e na Serra do Cipó (MG) (Haddad et al. 1988, Eterovick 2003, Eterovick \& Sazima 2004, Silveira 2006), na Floresta Nacional da Silvânia (GO) (Bastos et al. 2003), em Tocantins e no Maranhão (Brasileiro et al. 2008), em Guararapes, Rio Claro, Itirapina, Brotas e Assis (SP) (Bernarde \& Kokubum 1999, Toledo et al. 2003, Brasileiro et al. 2005, Bertoluci et al. 2007).
Trabalhos com comunidades de anuros do cerrado ainda são escassos, e a forte pressão antrópica, o alto grau de endemismo e o declínio de populações de anuros registrados no cerrado brasileiro reforçam a importância de inventários taxonômicos em áreas de cerrado. Apresentamos a seguir uma lista de espécies de anuros em duas unidades de conservação do cerrado do município de Assis (SP). Adicionalmente, apresentamos comentários sobre biologia, ecologia e distribuição geográfica das espécies registradas em nosso estudo. Este trabalho complementa os dados descritos por Bertoluci et al. (2007) para a mesma área.

\section{Material e Métodos}

\section{1. Área de estudo}

O presente estudo foi realizado no município de Assis, sudoeste do Estado de São Paulo, em duas unidades de conservação com áreas contínuas $\left(22^{\circ} 36^{\prime} \mathrm{S}\right.$ e $\left.50^{\circ} 23^{\prime} \mathrm{W}\right)$ : Estação Ecológica de Assis (EEA) e Floresta Estadual de Assis (FEA), em altitudes que variam entre 520 e $590 \mathrm{~m}$ (Figura 1). A EEA possui 1.760,64 ha, com fisionomia predominante de cerradão, mas apresenta também cerrado strictu sensu e tipos florestais ripários. A FEA possui 2.816,42 ha, onde predominam reflorestamentos com espécies vegetais exóticas (Pinus spp. e Eucalyptus spp.). Essas áreas encontram-se em zona de transição entre dois tipos climáticos, Cwa e Cfa de Köppen, ambos caracterizados por verões quentes e chuvosos e invernos secos, mas diferenciados pela duração do período seco, mais longo ou mais curto,
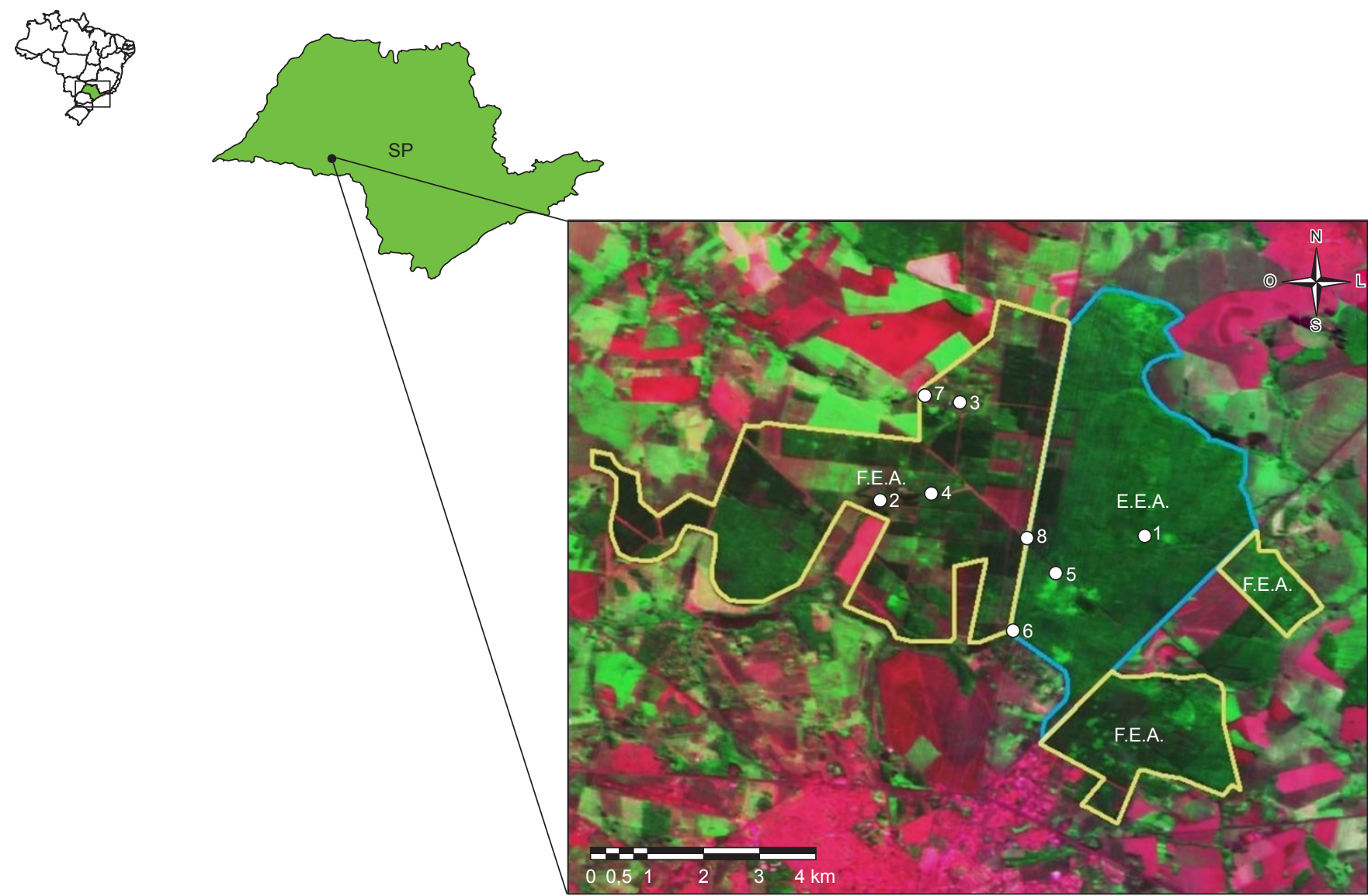

Figura 1. Localização da área de estudo e dos corpos d'água amostrados na EEA e na FEA (números 1 a 8), município de Assis, Estado de São Paulo, sudeste do Brasil.

Figure 1. Location of the study site and of the water bodies studied in the EEA and the FEA (numbered 1 to 8), municipality of Assis, State of São Paulo, southeastern Brazil. 
respectivamente, sendo que a precipitação anual média oscila em torno de $1.450 \mathrm{~mm}$, estando concentrada no período compreendido entre outubro e março.

\section{Coleta de dados}

Entre outubro de 2005 e outubro de 2007, oito corpos d'água previamente selecionados foram visitados mensalmente (dois dias consecutivos) por dois pesquisadores. As características desses corpos d'água, quatro permanentes e quatro temporários, estão mostradas na Tabela 1 e na Figura 2. Utilizamos as amostragens visual e auditiva simultaneamente, dois métodos considerados complementares (Crump \& Scott 1994, Zimmerman 1994) durante a noite (18:00-24:00 horas) por dois dias consecutivos por mês.

Tabela 1. Principais características dos oito corpos d'água estudados na EEA e na FEA: FP, floresta plantada com Pinus; VA, vegetação arbustiva esparsa (e.g. Melastomataceae, Mimosaceae, Anacardiaceae); VH, vegetação herbácea ereta (e.g. Poaceae, Cyperaceae); SN, solo nu. A sequiência de tipos de vegetação no item "Tipo de vegetação marginal” indicam sua predominância decrescente.

Table 1. Major features of the eight water bodies studied in EEA and FEA: FP, Pinus forest; VA, sparse bushes (e.g. Melastomataceae, Mimosaceae, Anacardiaceae); VH, herbaceous vegetation (e.g. Poaceae, Cyperaceae); SN, naked soil. The order of vegetation types under "Tipo de vegetação marginal" reflects the decreasing predominance of types.

\begin{tabular}{|c|c|c|c|c|}
\hline Corpos d'água & Localização geográfica & Hidroperíodo & Área $\left(\mathrm{m}^{2}\right)$ & Tipo de vegetação marginal \\
\hline 1 & $22^{\circ} 35^{\prime} 52^{\prime \prime}$ S e $50^{\circ} 22^{\prime} 19^{\prime \prime} \mathrm{W}$ & Permanente & 117 & Cerradão \\
\hline 2 & $22^{\circ} 35^{\prime} 27^{\prime \prime}$ S e $50^{\circ} 25^{\prime} 14^{\prime \prime} \mathrm{W}$ & Permanente & $>1000$ & FP, VA \\
\hline 3 & $22^{\circ} 34^{\prime} 21^{\prime \prime}$ S e $50^{\circ} 24^{\prime} 38^{\prime \prime} \mathrm{W}$ & Temporário & 338 & VH \\
\hline 4 & $22^{\circ} 35^{\prime} 23^{\prime \prime}$ S e $50^{\circ} 24^{\prime} 41^{\prime \prime} \mathrm{W}$ & Temporário & 49 & $\mathrm{VH}, \mathrm{VA}$ \\
\hline 5 & $22^{\circ} 35^{\prime} 59^{\prime \prime} \mathrm{S}$ e $50^{\circ} 23^{\prime} 40^{\prime \prime} \mathrm{W}$ & Temporário & 80 & SN, Cerradão, VH \\
\hline 6 & $22^{\circ} 36^{\prime} 42^{\prime \prime} \mathrm{S}$ e $50^{\circ} 23^{\prime} 57^{\prime \prime} \mathrm{W}$ & Permanente & $>1000$ & Cerradão \\
\hline 7 & $22^{\circ} 34^{\prime} 25^{\prime \prime} \mathrm{S}$ e $50^{\circ} 24^{\prime} 46^{\prime \prime} \mathrm{W}$ & Permanente & $>1000$ & VA, VH, SN \\
\hline 8 & $22^{\circ} 35^{\prime} 48^{\prime \prime} \mathrm{S}$ e $50^{\circ} 23^{\prime} 48^{\prime \prime} \mathrm{W}$ & Temporário & 104 & $\mathrm{VH}, \mathrm{FP}$ \\
\hline
\end{tabular}
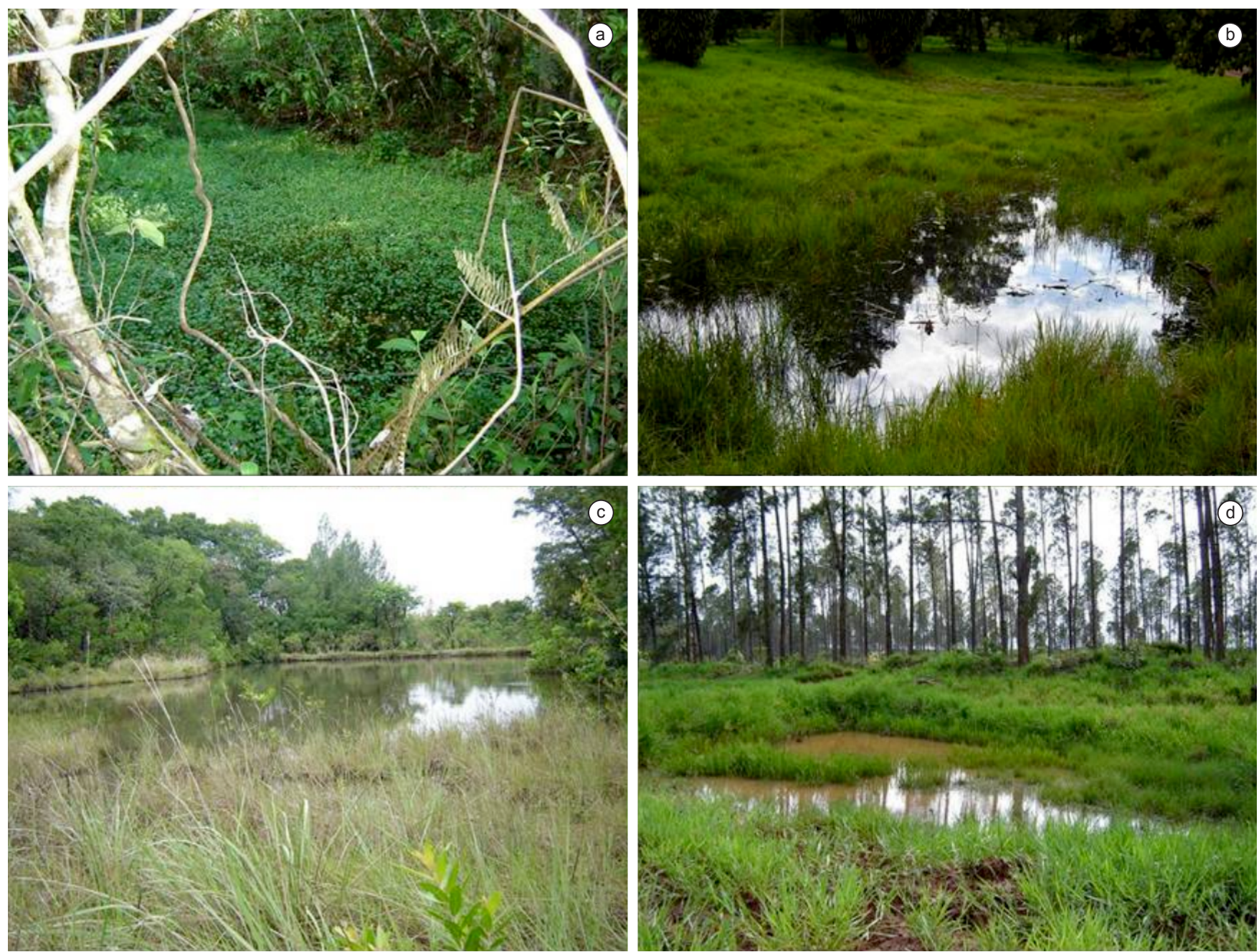

Figura 2. Quatro corpos d'água estudados na EEA e na FEA. a,c) permanentes, b,d) temporários.

Figure 2. Four water bodies searched for anurans in the EEA and the FEA. a,c) permanent, b,d) temporary. 
Entre outubro de 2005 e setembro 2006, foram feitas amostragens diurnas em uma parcela permanente de 320 × $320 \mathrm{~m}$. O método utilizado foi o de procura visual durante caminhadas por 16 transectos de $320 \mathrm{~m}$, quando os animais eram procurados pelo revolvimento da camada de serapilheira numa faixa de $1 \mathrm{~m}$ de largura. Os 16 transectos eram feitos em dois dias consecutivos. O esforço amostral total foi de 240 horas/pessoa. Todas as espécies encontradas durante o deslocamento entre as áreas de amostragem e as áreas adjacentes também foram registradas.

A eficiência da amostragem foi avaliada por curvas de rarefação construídas com dados de presença/ausência das espécies em cada mês de amostragem utilizando o Programa EstimateS v. 7.5.0 com 1.000 aleatorizações (Colwell 2005). O programa gera 1.000 curvas de acumulação de espécies, o que aleatoriza a ordem das amostras; assim, cada ponto da curva corresponde à média de riqueza acumulada nas 1.000 curvas. Cada amostra corresponde a uma viagem mensal, resultando em um total de 25 amostras. Curvas de rarefação, além de se constituírem em uma medida de riqueza de espécies, servem para comparar amostras com diferentes números de indivíduos (Gotelli \& Graves 1996). Para a estimativa da riqueza de espécies, foram utilizados os estimadores não-paramétricos Bootstrap e Jacknife 1.

A nomenclatura adotada neste trabalho está de acordo com Frost (2008). Pelo menos um indivíduo de cada espécie foi coletado, anestesiado até a morte por meio da inalação de $\mathrm{CO}_{2}$ (de acordo com a resolução $\mathrm{N}^{\circ}$. 714 de 20 de junho de 2002 da CFMV), fixado em formalina $10 \%$ e conservado em uma solução de álcool etílico a $70 \%$ (McDiarmid 1994), sendo então incorporado à coleção herpetológica do Laboratório de Zoologia de Vertebrados da ESALQ-USP (acrônimo VESALQ; licença de coleta IBAMA número 430/05).

\section{Resultados e Discussão}

Durante este estudo, foram registradas 23 espécies de anuros distribuídas em 13 gêneros e seis famílias: Hylidae (oito espécies), Leptodactylidae (seis espécies), Leiuperidae (quatro espécies), Microhylidae (três espécies), Bufonidae (uma espécie) e Cycloramphidae (uma espécie) (Tabela 2). Este inventário acrescentou quatro espécies aos dados descritos por Bertoluci et al. (2007) (Pseudopaludicola cf. mystacalis, Elachistocleis sp.2, Leptodactylus ocellatus e L. furnarius). Apresentamos a seguir breves comentários sobre cada espécie registrada nas duas áreas de estudo.

\section{BUFONIDAE}

\section{Rhinella schneideri (Figura 3a)}

Os machos vocalizam no nível da água, parcialmente submersos, em lagoas e represas perenes, locais onde os girinos se desenvolvem (Toledo et al. 2003). A espécie possui reprodução explosiva, frequentemente ocorrendo no final da estação seca e fria e no início da estação chuvosa e quente (Toledo et al. 2003, Brasileiro et al. 2005). Esse padrão de atividade reprodutiva é encontrado em outras espécies do gênero, como $R$. icterica e $R$. ornata (Bertoluci 1998, Bertoluci \& Rodrigues 2002a, Canelas \& Bertoluci 2007). A espécie é amplamente distribuída, sendo encontrada em vários habitats, incluindo o Chaco, o Cerrado e regiões de Mata Atlântica, embora comumente ocorra em áreas abertas e urbanizadas (IUCN 2006). Está distribuída da costa atlântica brasileira (do Ceará até o Rio Grande do Sul) até o Paraguai e parte central da Bolívia, de sudoeste a nordeste e parte central da Argentina e nordeste do Uruguai (Frost 2008).

\section{CYCLORAMPHIDAE}

\section{Odontophrynus americanus}

Essa espécie possui hábito semi-fossório e é encontrada durante a primavera e verão em poças temporárias, áreas alagáveis e lagoas de áreas abertas, onde os machos vocalizam à noite na borda e dentro da água (Cei 1980, Bertoluci 1998, Bertoluci \& Rodrigues 2002b, Conte \& Machado 2005). Os ovos são depositados no fundo de corpos d'água temporários, onde os girinos se desenvolvem (Cei 1980). Reproduz-se no final da estação seca e fria e início da estação quente e chuvosa (Bertoluci 1998, Conte \& Machado 2005). Ocorre em áreas abertas e savanas no centro-oeste, sul e sudeste brasileiro, Uruguai, Paraguai e no nordeste e parte central da Argentina, em altitudes que variam de 0 a $2.000 \mathrm{~m}$ (IUCN 2006).

\section{HYLIDAE}

\section{Dendropsophus minutus (Figura 3b)}

Os machos normalmente vocalizam empoleirados na vegetação emergente e marginal de corpos d'água temporários e permanentes localizados em áreas abertas, borda da mata e interior da floresta, entre 10 a $70 \mathrm{~cm}$ de altura (Bertoluci \& Rodrigues 2002b). Em grandes agregações, ocorre expansão do nicho estrutural, com os machos utilizando, além da vegetação marginal, a vegetação flutuante como plataforma de vocalização (Bertoluci \& Rodrigues 2002b). Como sugerido por Bertoluci \& Rodrigues (2002b), esse comportamento pode estar associado ao espaçamento entre os machos, que apresentam comportamento agressivo dirigido aos machos rivais (Cardoso \& Haddad 1984) e comportamento do macho-satélite (Haddad 1991). Os ovos são depositados diretamente na água, na forma de pequenos aglomerados ancorados à vegetação submersa (Bertoluci 1991). Em áreas de Mata Atlântica, os machos vocalizam ao longo de todo o ano (Bertoluci 1998, Bertoluci \& Rodrigues 2002a); em outros biomas ou áreas de transição, a reprodução está associada à estação quente e chuvosa (Brasileiro et al. 2005, Kopp \& Eterovick 2006, Canelas \& Bertoluci 2007). É uma das espécies de anfíbios mais comuns na América do Sul, ocorrendo em áreas de planície ao leste dos Andes, em altitudes de até 2.000 m (Frost 2008).

\section{Dendropsophus nanus (Figura 3c)}

Os machos vocalizam em poças de áreas abertas empoleirados em gramíneas ou vegetação arbustiva marginal ou sobre a água a até $80 \mathrm{~cm}$ de altura (Rossa-Feres \& Jim 2001, Toledo et al. 2003). Os ovos são ancorados às plantas aquáticas emergentes, e os girinos são noturnos, desenvolvendo-se nos mesmo corpo d'água (Cei 1980). A espécie se reproduz ao longo da estação quente e chuvosa (Toledo et al. 2003, Santos et al. 2007). Ocorre em quase todo o interior do Brasil, da bacia Amazônica até o Rio Grande do Sul, Paraguai, parte oriental da Bolívia, Uruguai e no norte e bacia do Prata, na Argentina (Frost 2008).

\section{Hypsiboas albopunctatus (Figura 3d)}

Os machos vocalizam à noite empoleirados na vegetação baixa na borda de corpos d'água lênticos permanentes e temporários, onde os ovos são depositados e os girinos se desenvolvem até a metamorfose (Toledo et al. 2003, Vasconcelos \& Rossa-Feres 2005). A reprodução ocorre principalmente ao longo da estação quente e chuvosa (Bertoluci \& Rodrigues 2002a, Toledo et al. 2003, Brasileiro et al. 2005, Canelas \& Bertoluci 2007, Santos et al. 2007). Sua distribuição geográfica inclui a parte central, sudeste e sudoeste do Brasil, além do nordeste da Argentina, leste da Bolívia e leste do Paraguai (Frost 2008).

\section{Hypsiboas faber (Figura 3e)}

A atividade reprodutiva ocorre durante a estação chuvosa (Bertoluci 1998, Bertoluci \& Rodrigues 2002a, Conte \& Machado 2005, Canelas \& Bertoluci 2007). Tipicamente, os machos 

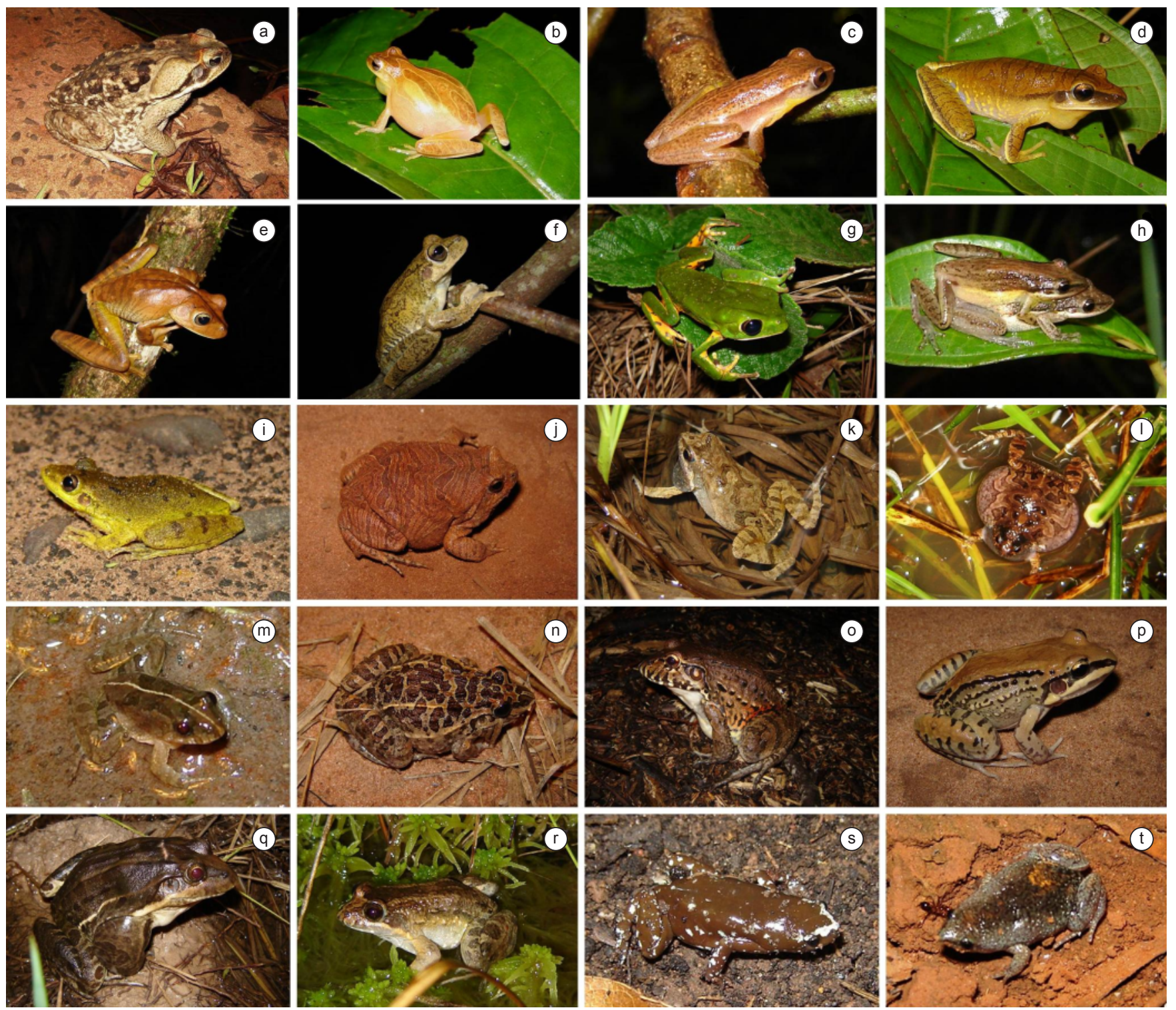

Figura 3. Espécies de anfíbios anuros encontradas na EEA e na FEA entre outubro de 2005 e outubro de 2007. a) Rhinella schneideri, b) Dendropsophus minutus, c) D. nanus, d) Hypsiboas albopunctatus, e) H. faber, f) H. lundii, g) Phyllomedusa tetraploidea, h) Scinax fuscomarginatus, i) S. fuscovarius, j) Eupemphix nattereri, k) Physalaemus cuvieri, 1) Physalaemus marmoratus; m) Pseudopaludicola cf. mystacalis, n) Leptodactylus fuscus, o) L. labyrinthicus, p) L. mystacinus, q) L. ocellatus, r) L. podicipinus, s) Chiasmocleis albopunctata e, t) Elachistocleis sp.2.

Figure 3. Anuran amphibian species recorded in the EEA and the FEA between October 2005 and October 2007. a) Rhinella schneideri, b) Dendropsophus minutus, c) D. nanus, d) Hypsiboas albopunctatus, e) H. faber, f) H. lundii, g) Phyllomedusa tetraploidea, h) Scinax fuscomarginatus, i) S. fuscovarius, j) Eupemphix nattereri, k) Physalaemus cuvieri, 1) Physalaemus marmoratus; m) Pseudopaludicola cf. mystacalis, n) Leptodactylus fuscus, o) L. labyrinthicus, p) L. mystacinus, q) L.ocellatus, r) L. podicipinus, s) Chiasmocleis albopunctata and, t) Elachistocleis sp.2.

vocalizam a partir do chão (Bertoluci \& Rodrigues 2002b), geralmente ao lado de ninhos escavados nas margens de corpos d'água permanentes e temporários, que defendem agressivamente de machos rivais (Martins \& Haddad 1988, Bertoluci 1991). Os girinos permanecem nos ninhos até que estes sejam inundados ou destruídos pela chuva (Martins \& Haddad 1988). A espécie tem distribuição ampla no sul da América do Sul, da Bahia ao Rio Grande do Sul, na província de Misiones na Argentina e sudeste do Paraguai (Frost 2008).

\section{Hypsiboas lundii (Figura 3f)}

Essa espécie pode ser encontrada em florestas primárias e secundárias, próximo de riachos, sendo totalmente dependente desse tipo de formação vegetal em todas as fases do seu ciclo de vida; os machos vocalizam na vegetação, de 1,5 a $10 \mathrm{~m}$ de altura (Brasileiro et al. 2005). Reproduz-se ao longo da estação chuvosa e esporadicamente na estação seca (Brasileiro et al. 2005). Ocorre nas áreas de cerrado dos estados de Minas Gerais, São Paulo e Goiás e do Distrito Federal (Frost 2008).

\section{Phyllomedusa tetraploidea (Figura 3g)}

Os machos vocalizam expostos em meio a moitas de gramíneas ou taboas, em brejos ou poças. A desova é depositada em folhas pendentes sobre a água; após a eclosão, os girinos caem na água, onde se desenvolvem até a metamorfose (Pombal \& Haddad 1992). A reprodução ocorre ao longo da estação quente e 
Tabela 2. Espécies de anuros encontradas na Estação Ecológica de Assis (EEA) e na Floresta Estadual de Assis (FEA), estado de São Paulo, sudeste do Brasil.

Table 2. Anuran species of the Estação Ecológica de Assis (EEA) and the Floresta Estadual de Assis (FEA), state of São Paulo, southeastern Brazil.

\begin{tabular}{|c|c|c|c|}
\hline & EEA & FEA & $\mathbf{N}^{\circ}$ de Campo (VESALQ) \\
\hline \multicolumn{4}{|l|}{ BUFONIDAE } \\
\hline Rhinella schneideri (Werner, 1984) & $\mathrm{x}$ & $\mathrm{x}$ & Assis 015 \\
\hline \multicolumn{4}{|l|}{ CYCLORAMPHIDAE } \\
\hline Odontophrynus americanus (Duméril \& Bibron, 1841) & $\mathrm{x}$ & - & Assis 016 \\
\hline \multicolumn{4}{|l|}{ HYLIDAE } \\
\hline Dendropsophus minutus (Peters, 1872) & $\mathrm{x}$ & $\mathrm{x}$ & Assis 007,013 \\
\hline Dendropsophus nanus (Boulenger, 1889) & $\mathrm{x}$ & $\mathrm{x}$ & Assis 008,027 \\
\hline Hypsiboas albopunctatus (Spix, 1824) & $\mathrm{x}$ & $\mathrm{x}$ & Assis 006 \\
\hline Hypsiboas faber (Wied-Neuwied, 1821) & $\mathrm{x}$ & $\mathrm{x}$ & Assis 022 \\
\hline Hypsiboas lundii (Burmeister, 1856) & $\mathrm{x}$ & $\mathrm{x}$ & Assis 005 \\
\hline Phyllomedusa tetraploidea Pombal \& Haddad, 1992 & $\mathrm{x}$ & & Assis 025 \\
\hline Scinax fuscomarginatus (A. Lutz, 1925) & $\mathrm{x}$ & $\mathrm{x}$ & Assis 010, 011, 019 \\
\hline Scinax fuscovarius (A. Lutz, 1925) & $\mathrm{x}$ & $\mathrm{x}$ & Assis 009 \\
\hline \multicolumn{4}{|l|}{ LEIUPERIDAE } \\
\hline Eupemphix nattereri Steindachner, 1863 & $\mathrm{x}$ & $\mathrm{x}$ & Assis 003 \\
\hline Physalaemus cuvieri Fitzinger, 1826 & $\mathrm{x}$ & $\mathrm{x}$ & Assis $004,017,026$ \\
\hline Physalaemus marmoratus (Reinhardt \& Lütken, 1862 “1861”) & - & $\mathrm{x}$ & Assis 002 \\
\hline Pseudopaludicola cf. mystacalis & - & $\mathrm{x}$ & Assis 028,029 \\
\hline \multicolumn{4}{|l|}{ LEPTODACTYLIDAE } \\
\hline Leptodactylus furnarius Sazima \& Bokermann, 1978 & $\mathrm{x}$ & $\mathrm{x}$ & Assis 030,032 \\
\hline Leptodactylus fuscus (Schneider, 1799) & $\mathrm{x}$ & $\mathrm{x}$ & Assis 001 \\
\hline Leptodactylus labyrinthicus (Spix, 1824) & $\mathrm{x}$ & $\mathrm{x}$ & Assis 020 \\
\hline Leptodactylus mystacinus (Burmeister, 1861) & $\mathrm{x}$ & $\mathrm{x}$ & Assis 014 \\
\hline Leptodactylus ocellatus (Linnaeus, 1758) & - & $\mathrm{x}$ & Assis 031 \\
\hline Leptodactylus podicipinus (Cope, 1862) & - & $\mathrm{x}$ & Assis 021,033 \\
\hline \multicolumn{4}{|l|}{ MICROHYLIDAE } \\
\hline Chiasmocleis albopunctata (Boettger, 1885) & $\mathrm{x}$ & $\mathrm{x}$ & Assis $012,018,034$ \\
\hline Elachistocleis sp.1 & $\mathrm{x}$ & $\mathrm{x}$ & Assis 024 \\
\hline Elachistocleis sp.2 & $\mathrm{x}$ & $\mathrm{x}$ & Assis 023 \\
\hline
\end{tabular}

chuvosa (Bernarde \& Anjos 1999). Existe registro de hibridação com a espécie Phyllomedusa distincta na área de contato entre as duas espécies (Ribeirão Branco, SP); nessa área, ocorrem triplóides viáveis, porém estéreis (Pombal \& Haddad 1992). Ocorre no interior dos estados de São Paulo e Paraná, na província de Misiones na Argentina e região sudeste do Paraguai, em altitudes que variam de 400 a 1.000 m (IUCN 2006).

\section{Scinax fuscomarginatus (Figura 3h)}

Os machos vocalizam nas margens de lagoas temporárias ou permanentes, usando a vegetação marginal como poleiro (Toledo \& Haddad 2005b, Vasconcelos \& Rossa-Feres 2005). Os ovos são depositados diretamente na água, onde os girinos se desenvolvem até a metamorfose (Bastos et al. 2003, Toledo \& Haddad 2005a). A reprodução ocorre na estação quente e chuvosa (Brasileiro et al. 2005). A espécie distribui-se amplamente no interior do Brasil, parte oriental da Bolívia, Paraguai e nordeste da Argentina (Frost 2008).
8. Scinax fuscovarius (Figura 3i).

Espécie comumente invasora de habitats alterados (Bertoluci \& Heyer 1995), reproduz-se predominantemente em corpos d'água temporários de áreas abertas (Bertoluci \& Rodrigues 2002b, Santos et al. 2007), Os machos vocalizam nas margens desses corpos d'água, no chão ou sobre a vegetação marginal (até $40 \mathrm{~cm}$ de altura) (Rossa-Feres \& Jim 2001, Bertoluci \& Rodrigues 2002b, Brasileiro et al. 2005). Os ovos são depositados na vegetação aquática ou diretamente na água (Rodrigues et al. 2005). O período reprodutivo estende-se durante toda a estação quente e chuvosa (Toledo et al. 2003, Brasileiro et al. 2005, Rodrigues et al. 2005, Santos et al. 2007), mas grandes agregações no início da estação e evidências de reprodução explosiva já foram relatadas em localidades de Mata Atlântica (Bertoluci 1998, Bertoluci \& Rodrigues 2002a). Essa espécie é amplamente distribuída em ambientes abertos do complexo Cerrado-Caatinga-Chaco (Duellman 1999), abrangendo o sudeste do Brasil, o nordeste da Argentina, o Paraguai e a Bolívia, em altitudes que variam de 150 a 1.800 m (Frost 2008). 


\section{LEIUPERIDAE}

\section{Eupemphix nattereri (Figura 3j)}

Os machos vocalizam flutuando ou parcialmente submersos em áreas com plantas herbáceas e gramíneas e em concavidades no barranco da margem de corpos d'água permanentes e temporários (Bernarde \& Kokubum 1999, Rossa-Feres \& Jim 2001). O casal em amplexo constrói ninhos de espuma flutuantes, que evitam o dessecamento dos ovos em poças temporárias; após a eclosão, os girinos abandonam o ninho e completam o desenvolvimento na água (Cei 1980). Os machos vocalizam caracteristicamente depois de chuvas fortes ao longo da estação chuvosa (Brasileiro et al. 2005). Essa espécie possui distribuição ampla na parte central e sudeste do Brasil e na parte oriental da Bolívia e do Paraguai (Frost 2008).

\section{Physalaemus cuvieri (Figura 3k)}

Os machos vocalizam nas margens de corpos d'água temporários e semipermanentes, ao nível do chão, frequentemente escondidos na base vegetação marginal e emergente (Bertoluci 1991, Rossa-Feres \& Jim 2001, Bertoluci \& Rodrigues 2002b, Toledo et al. 2003). Durante o amplexo, o casal constrói um ninho de espuma flutuante, que é ancorado à vegetação aquática e contém ovos totalmente despigmentados (Cei 1980, Bertoluci 1991). A reprodução ocorre ao longo da estação quente e chuvosa (Bertoluci 1998, Bertoluci \& Rodrigues 2002a, Toledo et al. 2003, Brasileiro et al. 2005, Canelas \& Bertoluci 2007). Essa espécie ocorre amplamente no Brasil, sendo encontrada desde o Rio Grande do Sul até a região amazônica, parte oriental do Paraguai, Argentina e Departamento de Santa Cruz, na Bolívia (Frost 2008).

\section{Physalaemus marmoratus (Figura 31)}

P. marmoratus se reproduz em poças temporárias de áreas abertas, onde o casal em amplexo deposita ovos despigmentados em ninhos de espuma flutuantes (Nascimento et al. 2006). Essa espécie ocorre em áreas abertas dos estados da Bahia, Espírito Santo, Rio de Janeiro, São Paulo, Minas Gerais, Goiás, Mato Grosso e Mato Grosso do Sul e em áreas adjacentes do Paraguai e Bolívia (Frost 2008).

\section{Pseudopaludicola cf. mystacalis (Figura 3m)}

A identificação das espécies do gênero Pseudopaludicola é problemática, sendo comuns erros de determinação (Lobo 1992, 1994). A história natural das espécies desse gênero é pouco conhecida (Lynch 1989, Lobo 1992). Em Assis, foram observados machos vocalizando sempre no início da noite em áreas paludosas de ambientes permanentes e temporários.

\section{LEPTODACTYLIDAE}

\section{Leptodactylus furnarius}

Os machos escavam tocas nas margens de lagoas temporárias e locais brejosos, onde seu ninho de espuma é depositado (Brasileiro et al. 2005). Reproduz-se no meio da estação chuvosa e quente (Brasileiro et al. 2005). Essa espécie ocorre no sudeste, sul e parte central do Brasil, Uruguai, Paraguai e Argentina (Frost 2008, Baldo et al. 2008).

\section{Leptodactylus fuscus (Figura 3n)}

Os machos vocalizam e constroem tocas nas margens de lagoas temporárias de áreas abertas (Cei 1980, Rossa-Feres \& Jim 2001, Bertoluci \& Rodrigues 2002b, Brasileiro et al. 2005). Os ovos são depositados na forma de ninhos de espuma dentro da toca, onde os girinos passam pelos primeiros estágios de desenvolvimento, que se completa no corpo d'água principal (Toledo et al. 2003). O período reprodutivo está associado à estação chuvosa (Bertoluci 1998, Bernarde \& Kokubum 1999, Vasconcelos \& Rossa-Feres 2005). Essa espécie tem ampla distribuição na América do Sul, ocorrendo nas savanas do Panamá e em todo o leste dos Andes (Frost 2008).

\section{Leptodactylus labyrinthicus (Figura 3o)}

Os machos vocalizam em lagoas temporárias, riachos e lagoas permanentes, podendo utilizar, como sítios de vocalização, buracos e depressões do chão ou até mesmo águas rasas, mantendo sempre a cabeça fora da água. O casal em amplexo deposita os ovos em ninhos de espuma conspícuos na borda de lagoas temporárias, quase sempre cobertos ou circundados pela vegetação (Zina \& Haddad 2005). A reprodução está associada ao período mais quente e chuvoso do ano (Brasileiro et al. 2005, Vasconcelos \& Rossa-Feres 2005). Amplamente distribuída no Brasil, essa espécie pode ser encontrada nos cerrados e caatingas de Roraima, Rondônia, Amapá, Pará, regiões nordeste, sudeste e central do Brasil, costa da Venezuela, parte oriental do Paraguai, Bolívia e norte da Argentina (IUCN 2006).

\section{Leptodactylus mystacinus (Figura 3p)}

Os machos vocalizam no chão, às margens de corpos d'água temporários. Nos primeiros estágios, os ovos e os girinos se desenvolvem em ninhos de espuma depositados na toca subterrânea construída pelo macho, completando seu desenvolvimento no corpo d'água adjacente, como em L. fuscus (Toledo et al. 2003, Brasileiro et al. 2005). A reprodução ocorre do final da estação seca ao final da estação chuvosa (Bernarde \& Kokubum 1999, Toledo et al. 2003, Brasileiro et al. 2005). A espécie ocorre no sudeste da Bolívia e parte oriental do Brasil até Uruguai, Paraguai e Argentina (Frost 2008).

\section{Leptodactylus ocellatus (Figura 3q)}

Os machos de L. ocellatus vocalizam ao nível d'água em ambientes aquáticos localizados em áreas abertas ou na borda da mata (Bertoluci \& Rodrigues 2002b). Os ovos são depositados em ninhos de espuma flutuantes e a fêmea permanece com os ovos e girinos, protegendo-os de predadores (Vaz-Ferreira \& Gehrau 1975, Bertoluci 1991). Os girinos dessa espécie são gregários, deslocandose em cardumes globulares (Cei 1980, Bertoluci \& Rodrigues 2002b). A reprodução ocorre ao longo da estação quente e chuvosa (Bertoluci 1998, Bertoluci \& Rodrigues 2002a, Brasileiro et al. 2005, Conte \& Rossa-Feres 2006, Canelas \& Bertoluci 2007). Ocorre em toda a América do Sul ao leste dos Andes (Frost 2008).

\section{Leptodactylus podicipinus (Figura 3r)}

Habita áreas ripárias, vivendo e se reproduzindo entre as plantas e raízes que cobrem as margens de rios e lagoas temporárias e permanentes (Cei 1980, Santos et al. 2007). Os ovos são depositados em águas rasas, onde as larvas desenvolvem-se até a metamorfose (Cei 1980). Os machos podem vocalizar ao longo de todo o ano, com pico de reprodução na estação chuvosa (Bernarde \& Kokubum 1999, Santos et al. 2007). Ocorre em formações abertas do Paraguai ao sul do Uruguai, Argentina, Bolívia, algumas áreas do sudeste, sul, parte central do Brasil e ao longo dos rios Madeira e Amazonas, em altitudes maiores que $550 \mathrm{~m}$ (Frost 2008).

\section{MICROHYLIDAE}

\section{Chiasmocleis albopunctata (Figura 3s)}

Grandes agregações de machos vocalizando, com o corpo parcialmente submerso, podem ser encontradas em corpos d'água 
temporários e em pequenas poças formadas por chuvas fortes, ao longo da estação chuvosa (Toledo et al. 2003, Brasileiro et al. 2005). Os ovos são depositados em corpos d'água lênticos, onde os girinos se desenvolvem (Toledo et al. 2003). Ocorre na parte leste da Bolívia, Paraguai e, no Brasil, nos estados de Goiás, Mato Grosso, Mato Grosso do Sul, Minas Gerais e São Paulo, em altitudes compreendidas entre 100 e 1.500 m (Frost 2008).

\section{Elachistocleis sp.1}

\section{Elachistocleis sp.2 (Figura 3t)}

A duas espécies acima fazem parte de uma confusão taxonômica que data de 1841 (Lavilla et al. 2003). Achamos conveniente não atribuir aos nossos espécimes nomes conhecidos até que o gênero sofra revisão, pois o fato de ocorrerem em sintopia dificulta a identificação (Kwet \& Di Bernardo 1998, Lavilla et al. 2003, Frost 2008). Todas as espécies desse gênero para as quais há dados publicados se reproduzem em poças temporárias de áreas abertas ou da borda da mata (Kwet \& Di Bernardo 1999, Rodrigues et al. 2003, Vasconcelos \& Rossa-Feres 2005, Thomé \& Brasileiro 2007).

A riqueza de espécies e a representatividade das famílias de anuros amostradas no cerrado da EEA e da FEA são similares às encontradas em outras áreas de cerrado do Brasil (Bastos et al. 2003, Brasileiro et al. 2005) e de formações vegetais antropizadas, com perda de área florestal e aumento de área aberta, como a Floresta Estacional Semidecidual (Toledo et al. 2003, Vasconcelos \& Rossa-Feres 2005, Santos et al. 2007), a Floresta Ombrófila Densa (Munduruca 2005) e a Floresta Ombrófila Mista (Conte \& Machado 2005).

A anurofauna de Assis é fortemente dominada pelas famílias Hylidae e Leptodactylidae, um padrão comumente encontrado nas regiões neotropicais (Aichinger 1989, Brasileiro et al. 2005, Kopp \& Eterovick 2006, Bertoluci \& Rodrigues 2002a, b, Bertoluci et al. 2007, Canelas \& Bertoluci 2007). A taxocenose de anuros da localidade de estudo é composta por espécies amplamente distribuídas em ambientes abertos do complexo Cerrado-Caatinga-Chaco (sensu Duellman 1999). Apenas Hypsiboas lundii é totalmente dependente da floresta de galeria em todas as fases do seu ciclo de vida (Brasileiro et al. 2005).

A curva de rarefação elaborada com dados das espécies encontradas em cada mês de coleta apresentou tendência de estabilização (Figura 4), indicando que a taxocenose foi amostrada quase que na sua totalidade. A riqueza estimada foi de 24,26 (Bootstrap) e de 25,88 espécies (Jacknife 1), o que sugere que poderia haver mais duas ou três espécies na área de estudo. Essa previsão já foi confirmada pelo encontro de duas espécies não registradas em nosso inventário durante o estudo feito para o plano de manejo da EEA (Cybele Araújo com. pess.).

Nenhuma espécie registrada nas duas áreas de estudo encontrase na lista de espécies ameaçadas do Estado de São Paulo (SMA 2009), do Brasil (Machado et al. 2005) ou da IUCN (Stuart et al. 2008). Esse fato, contudo, não reduz a importância dessas unidades de conservação, pois abrigam populações de espécies do Cerrado, um ambiente sujeito a forte pressão antrópica na forma de contaminação por agrotóxicos, destruição do habitat e desmatamento (Machado et al. 2005).

\section{Agradecimentos}

Os autores agradecem à FAPESP pelo apoio financeiro (processos 04/15938-5 e 99/09635-0), ao CNPq pela bolsa de iniciação científica concedida a JWRJ e de Produtividade em Pesquisa concedida a JB, a Vanessa K. Verdade pela ajuda na identificação das espécies, ao IBAMA pela licença de coleta ( $\left.{ }^{\circ} 430 / 05\right)$, ao Instituto Florestal de São Paulo pela permissão de acesso às duas unidades de conservação, e aos biólogos Ricardo A. Brassaloti, Henrique O. Sawakuchi, Vívian M. F. N. Vilela, Paula C. Lopes, Bruno H. C. Cavichioli, Gabriel L. Brejão, Mariana P. da Silva e Fernanda E. Nascimento pelo auxílio nos trabalhos de campo. Agradecemos especialmente a Ricardo A. Brassaloti pela ajuda com a identificação das espécies e com a confecção das curvas de rarefação e de estimativas de riqueza.

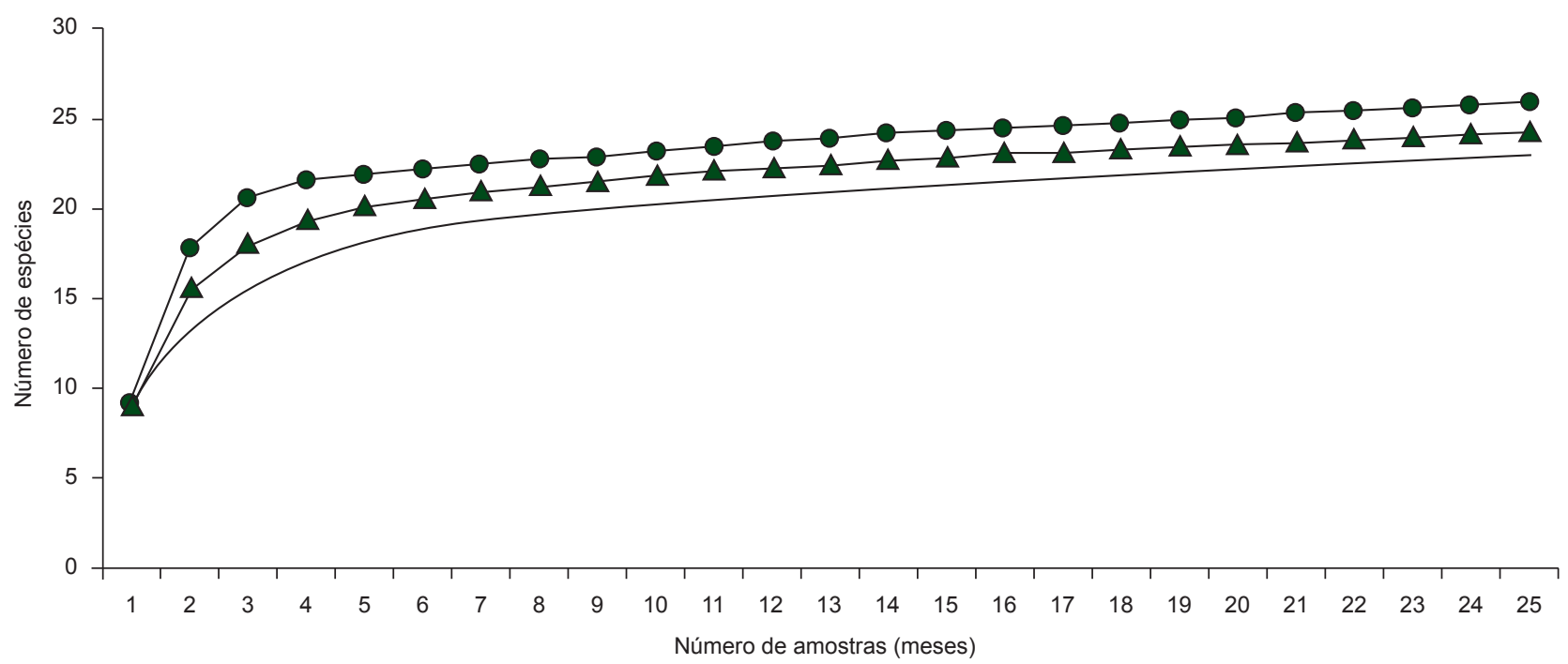

Figura 4. Curva de rarefação representando a riqueza cumulativa de espécies e curvas de riqueza estimada geradas pelos estimadores Bootstrap ( $\mathbf{\Delta}$ ) e Jacknife 1 (C) (Colwell 2005) ao longo de 25 meses (outubro de 2005 a outubro de 2007) conjuntamente na EEA e na FEA, SP. Curvas geradas com 1000 aleatorizações.

Figure 4. Rarefaction curve representing cumulative species richness and estimated richness curves generated by the estimators Bootstrap ( $\mathbf{\Delta}$ ) and Jacknife I

(C) (Colwell 2005) during 25 months (October 2005 to October 2007) in EEA and FEA. Curves were created by means of 1000 randomizations. 


\section{Referências Bibliográficas}

AICHINGER, M. 1987. Annual activity patterns of anurans in a seasonal neotropical environment. Oecologia, 71(4):583-592.

BALDO, D., TOMATIS, C. \& SEGALlA, M.V. 2008. Amphibia, Anura, Leptodactylidae, Leptodactylus furnarius: new country record, geographic distribution map and advertisement call. Check List, 4(2):98-102.

BASTOS, R.P., MOTTA, J.A.O., LIMA, L.P. \& GIMARÃES, L.D. 2003. Anfíbios da Floresta Nacional da Silvânia, Estado de Goiás. Stylo Gráfica e Editora, Goiânia.

BERNARDE, P.S. \& ANJOS, L. 1999. Distribuição espacial e temporal da anurofauna do Parque Estadual da Mata Godoy, Londrina, Paraná, Brasil (Amphibia: Anura). Comun. Mus. Ciênc. Tecnol. PUCRS, sér. Zool. 12:127-140.

BERNARDE, P.S. \& KOKUBUM, M.N.C. 1999. Anurofauna do município de Guararapes, estado de São Paulo, Brasil (Amphibia:Anura). Acta Biol. Leopoldensia, 21(1):89-97.

BERTOLUCI, J. 1991. Partição de recursos associada à atividade reprodutiva em uma comunidade de anuros (Amphibia) de Mata Atlântica. Dissertação de Mestrado, Universidade de São Paulo, São Paulo.

BERTOLUCI, J. 1998. Annual patterns of breeding activity in Atlantic Rainforest anurans. J. Herpetol. 32(4):607-611.

BERTOLUCI, J. \& HEYER, W.R. 1995. Boracéia Update. Froglog, 14:2-3.

BERTOLUCI, J. \& RODRIGUES, M.T. 2002a. Seasonal patterns of breeding activity of Atlantic rainforest anurans at Boracéia, southeastern Brazil. Amphibia-Reptilia, 23(2):161-167.

BERTOLUCI, J. \& RODRIGUES, M.T. 2002b. Utilização de hábitats reprodutivos e micro-habitats de vocalização em uma taxocenose de anuros (Amphibia) da Mata Atlântica do sudeste do Brasil. Pap. avulsos zool. S. Paulo, 42(11):287-297.

BERTOLUCI, J., BRASSALOTI, R.A., RIBEIRO Jr., J.W., VILELA, V.M.F.N. \& SAWAKUCHI, H.O. 2007. Species composition and similarities among anuran assemblages of forest sites in southeastern Brazil. Sci. agric. 64(4):364-374.

BITENCOURT, M.D. \& MENDONÇA, R.R. 2004. Viabilidade de conservação dos remanescentes de cerrado no estado de São Paulo. Annablume, São Paulo.

BRASILEIRO, C.A., SAWAYA, R.J., KIEFER, M.C. \& MARTINS, M 2005. Amphibians of an open cerrado fragment in southeastern Brazil. Biota Neotropica 5(2): http://www.biotaneotropica.org.br/v5n2/pt/ abstract?article+BN00405022005. (último acesso em 10/07/2008.)

BRASILEIRO, C.A., LUCAS, L.M., OYAMAGUCHI, H.M., THOMÉ, M.T.C. \& DIXO, M. 2008. Anurans, northern Tocantins River Basin, states of Tocantins and Maranhão, Brazil. Check List, 4(2):185-197.

CANELAS, M.A.S. \& BERTOLUCI, J. 2007. Anurans of the Serra do Caraça, southeastern Brazil: species composition and phenological patterns of calling activity. Iheringia, S. Zool. 97(1):21-26.

CARDOSO, A.J. \& HADDAD, C.F.B. 1984. Variabilidade acústica em diferentes populações e interações agressivas de Hyla minuta (Amphibia, Anura). Ciên. Cult. 36(8):1393-1399.

CEI, J.M. 1980. Amphibians of Argentina. Monitore Zool. Ital. (N.S) Monogr. 2:1-609.

COLWELL, R.K. 2005. EstimateS: statistical estimation of species richness and shared species from samples. Versão 7.5.2. Available from: $<$ http:// viceroy.eeb.uconn.edu/estimates $>$. (último acesso em 9/12/2008.)

CONTE, C.E. \& MACHADO, R.A. 2005. Riqueza de espécies e distribuição espacial e temporal em comunidades de anuros (Amphibia, Anura) em uma localidade de Tijucas do Sul, Paraná, Brasil. Rev. bras. zool. 22(4):940-948.

CONTE, C.E. \& ROSSA-FERES, D.C. 2006. Diversidade e ocorrência temporal da anurofauna (Amphibia, Anura) em São José dos Pinhais, Paraná, Brasil. Rev. bras. zool. 23(1):162-175.

CRUMP, M.L. \& SCOTT, N.J. 1994. Visual encounter surveys. In Measuring and Monitoring Biological Diversity: Standard Methods for Amphibians
(W.R. Heyer, M.A. Donnelly, R.W. McDiarmid, L.A.C. Hayek \& M.S Foster, eds.). Smithsonian Institution Press, Washington, p. 84-92.

DUELLMAN, W.E. 1999. Patterns of Distribution of Amphibians: a global perspective. The Johns Hopkins University Press, Baltimore.

ETEROVICK, P.C. 2003. Distribution of anuran species among montane streams in southeastern Brazil. J. Trop. Ecol. 19(3):219-228.

ETEROVICK, P.C. \& SAZIMA, I. 2004. Anfíbios da Serra do Cipó, Minas Gerais, Brasil. PUC Minas, Belo Horizonte.

FROST, D. 2008. Amphibian Species of the World: an Online Reference American Museum of Natural History, New York. Version 5.1. Available from: <http://research.amnh.org/herpetology/amphibia/index.php>. (último acesso em 8/12/2008.)

GOTELLI, N.J. \& GRAVES, G.R. 1996. Null Model in Ecology. Smithsonian Institutions Press, Washington D.C.

HADDAD, C.F.B., ANDRADE, G.V. \& CARDOSO, A.J. 1988. Anfíbios anuros do Parque Nacional da Serra da Canastra, Estado de Minas Gerais. Bras. Florest. 64(1):9-20.

IUCN, CONSERVATION INTERNATIONAL \& NATURESERVE. 2006. Global Amphibian Assessment. Available from: <http://www globalamphibians.org $>$. (último acesso em 10/07/2008.)

KLINK, C.A. \& MACHADO, R.B. 2005. Conservation of the Brazilian cerrado. Conserv. Biol. 19(3):707-713.

KOPP, K. \& ETEROVICK, P.C. 2006. Factors influencing spatial and temporal structure of frog assemblages at ponds in Southeastern Brazil. J. Nat. Hist. 40(29-31):1813-1830.

KWET, A. \& DI-BERNARDO, M. 1998. Elachistocleis erythrogaster, a new microhylid species from Rio Grande do Sul, Brazil. Stud. Neotrop Fauna \& Environm. 33(1):7-18.

KWET, A. \& DI-BERNARDO, M. 1999. Anfíbios. Amphibien. Amphibians. EDIPUCRS, Porto Alegre.

LAVILLA, E.O., VAIRA, M. \& FERRARI, L. 2003. A new species of Elachistocleis (Anura: Microhylidae) from the Andean Yungas of Argentina, with comments on the Elachistocleis ovalis-E. bicolor controversy. Amphibia-Reptilia, 24(3):269-284.

LOBO, F. 1992. Distribución y lista de localidades de Pseudopaludicola (Anura: Leptodactylidae) en la República Argentina. Cuad. Herp. 7(5):30-37.

LOBO, F. 1994. Descripción de una nueva especie de Pseudopaludicola (Anura: Leptodactylidae), redescripción de P. falcipes (Hensel, 1867) y $P$. saltica (Cope, 1887) y osteología de las tres especies. Cuad. Herp. 8(2):177-199.

LYNCH, J.D. 1989. A review of the leptodactylid frogs of the genus Pseudopaludicola in northen South America. Copeia, 1989(3):577-588.

MACHADO, A.B.M., MARTINS, C.S. \& DRUMMOND, G.M. (eds.). 2005 Lista da fauna brasileira ameaçada de extinção. Fundação Biodiversitas, Belo Horizonte.

MARTINS, M. \& HADDAD, C.F.B. 1988. Vocalization and reproductive behaviour in the smith frog, Hyla faber Wied (Amphibia: Hylidae) Amphibia-Reptilia, 9(1):49-60.

McDIARMID, R.W. 1994. Data standards. In Measuring and Monitoring Biological Diversity: Standard Methods for Amphibians. (W.R. Heyer, M.A. Donnelly, R.W. McDiarmid, L.A.C. Hayek \& M.S. Foster, eds.) Smithsonian Institution Press, Washington, p. 57-60.

MUNDURUCA, J.F.V. 2005. Gradientes ambientais e composição da comunidade de anuros da reserva Camurujipe, mata de São João, Bahia, Brasil. Dissertação de Mestrado, Universidade Federal da Bahia, Salvador.

MYERS, N., MITTERMEIER, R.A., MITTERMEIER, C.G., FONSECA, G.A.B. \& KENT, J. 2005. Biodiversity hotspots for conservation priorities. Nature, 403:853-858.

NASCIMENTO, L.B., PIMENTA, B.V.S., CRUZ, C.A.G. \& CARAMASCHI, U. 2006. Taxonomic status of Gomphobates marmoratus Reinhardt and Lutken, 1862 "1861" and Eupemphix fuscomaculatus Steindachner, 1864 (Amphibia, Anura, Leptodactylidae). S. Am. J. Herpetol. 1(3):166-174. 
POMBAL Jr., J.P. \& HADDAD, C.F.B. 1992. Espécies de Phyllomedusa do grupo burmeisteri do Brasil oriental, com descrição de uma espécie nova (Amphibia, Hylidae). Rev. bras. biol. 52(2):217-229.

RATTER, J.A., RIBEIRO, J.F. \& BRIDGEWATER, S. 1997. The Brazilian cerrado vegetation and threats to its biodiversity. Ann. Bot. 80(3):223-230

RODRIGUES, D.J., LOPES, F.S. \& UETANABARO, M. 2003. Padrão reprodutivo de Elachistocleis bicolor (Anura, Microhylidae) na Serra da Bodoquena, Mato Grosso do Sul, Brasil. Iheringia, Ser. Zool. 93(4):365-371.

RODRIGUES, D.J., UETANABARO, M. \& LOPES, F.S. 2005. Reproductive patterns of Trachycephalus venulosus (Laurenti, 1768) and Scinax fuscovarius (Lutz, 1925) from the Cerrado, Central Brazil. J. Nat. Hist. 39(35):3217-3226.

ROSSA-FERES, D.C. \& JIM, J. 2001. Similaridade do sítio de vocalização em uma comunidade de anfíbios anuros na região noroeste do Estado de São Paulo, Brasil. Rev. bras. zool. 18(2):439-454.

SANTOS, T.G., ROSSA-FERES, D.C. \& CASATTI, L. 2007. Diversidade e distribuição espaço-temporal de anuros em região com pronunciada estação seca do sudeste do Brasil. Iheringia, Ser. Zool. 97(1):37-49.

SILVEIRA, A.L. 2006. Anfíbios do município de João Pinheiro, uma área de cerrado no noroeste de Minas Gerais, Brasil. Arq. Mus. Nac. 64(2):131-139.

SECRETARIA DO MEIO AMBIENTE DO ESTADO DE SÃO PAULO SMA. 2009. Lista da fauna ameaçada de extinção no estado de São Paulo. Available from: <http://www.ambiente.sp.gov.br/fauna.php>. (último acesso em 04/02/2009.)

STUART, S., HOFFMAN, M., CHANSON, J., COX, N., BERRIDGE, R., RAMANI, P. \& YOUNG, B. (eds.). 2008. Threatened Amphibians of the World. Lynx Editions, Barcelona; IUCN, Gland. Switzerland \& Conservation International, Arlington.

TOLEDO, L.F. \& HADDAD, C.F.B. 2005a. Reproductive biology of Scinax fuscomarginatus (Anura, Hylidae) in south-eastern Brazil. J. Nat. Hist. 39(32):3029-3037.
TOLEDO, L.F. \& HADDAD, C.F.B. 2005b. Acoustic repertoire and calling behavior of Scinax fuscomarginatus (Anura, Hylidae). J. Herpetol. 39(3):455-464.

TOLEDO, L.F., ZINA, J. \& HADDAD, C.F.B. 2003. Distribuição espacial e temporal de uma comunidade de anfíbios anuros do Município de Rio Claro, São Paulo, Brasil. Holos Envir. 3(2):136-149.

THOMÉ, M.T.C. \& BRASILEIRO, C.A. 2007. Dimorfismo sexual, uso do ambiente e abundância sazonal de Elachistocleis cf. ovalis (Anura: Microhylidae) em um remanescente de Cerrado no estado de São Paulo, sudeste do Brasil. Biota Neotropica 7(1): http://www.biotaneotropica. org.br/v7n1/pt/abstract?article+bn00307012007. (último acesso em $10 / 12 / 2008$.)

VASCONCELOS, T.S. \& ROSSA-FERES, D.C. 2005. Diversidade, distribuição espacial e temporal de anfíbios anuros (Amphibia, Anura) na região noroeste do estado de São Paulo, Brasil. Biota Neotropica 5(2): http:// www.biotaneotropica.org.br/v5n2/pt/abstract?article+BN01705022005. (último acesso em 08/07/2008.)

VAZ-FERREIRA, R. \& GEHRAU, A. 1975. Comportamiento epimeletico de la rana comun, Leptodactylus ocellatus (L.) (Amphibia, Leptodactylidae). I. Atencion de la cria y actividades alimentarias y agresivas relacionados. Physis, 34(1):1-14.

ZIMMERMAN, B.L. 1994. Audio Strip Transects. In Measuring and Monitoring Biological Diversity: Standard Methods for Amphibians (W.R. Heyer, M.A. Donnelly, R.W. McDiarmid, L.A.C. Hayek \& M.S. Foster, eds.). Smithsonian Institution Press, Washington, p. 92-97.

ZINA, J. \& HADDAD, C.F.B. 2005. Reproductive activity and vocalizations of Leptodactylus labyrinthicus (Anura: Leptodactylidae) in southeastern Brazil. Biota Neotropica 5(2): http://www.biotaneotropica.org.br/v5n2/pt/ abstract?article+BN00605022005. (último acesso em 11/07/2008.)

Recebido em 31/10/08 Versão Reformulada recebida em 10/01/09 Publicado em 12/03/09 